ARTICLE OPEN

\title{
Ethnic-specific association of amylase gene copy number with adiposity traits in a large Middle Eastern biobank
}

\author{
Niccolo' Rossi ${ }^{1,5}$, Elbay Aliyev $\mathbb{D}^{2,5}$, Alessia Visconti ${ }^{1}{ }^{1}$, Ammira S. A. Akil ${ }^{2}$, Najeeb Syed ${ }^{2}$, Waleed Aamer (D) $^{2}$, Sujitha S. Padmajeya ${ }^{2}$,
} Mario Falchi ${ }^{1,6}$ and Khalid A. Fakhro iD $^{2,3,4,6 \bowtie}$

Studies assessing the impact of amylase genes copy number (CN) on adiposity report conflicting findings in different global populations, likely reflecting the impact of ancestral and ethnic-specific environment and lifestyle on selection at the amylase loci. Here, we leverage population size and detailed adiposity measures from a large population biobank to resolve confounding effects and determine the relationship between salivary (AMY1) and pancreatic (AMY2A) amylase genes CN and adiposity in 2935 Qatari individuals who underwent whole-genome sequencing (WGS) as part of the Qatar Genome Programme. We observe a negative association between $A M Y 1$ CNs and trunk fat percentage in the Qatari population $\left(P=7.50 \times 10^{-3}\right)$ and show that Qataris of Arab descent have significantly lower $\mathrm{CN}$ at $A M Y 1\left(P=1.32 \times 10^{-10}\right)$ as well as less favorable adiposity and metabolic profiles $(P<1.34 \times$ $10^{-8}$ ) than Qataris with Persian ancestry. Indeed, lower AMY1 CN was associated with increased total and trunk fat percentages in Arabs $\left(P<4.60 \times 10^{-3}\right)$ but not in Persians. Notably, overweight and obese Persians reported a significant trend towards dietary restraint following weight gain compared to Arabs $\left(P=4.29 \times 10^{-5}\right)$, with $A M Y 1 \mathrm{CN}$ showing negative association with dietary selfrestraint $\left(P=3.22 \times 10^{-3}\right)$. This study reports an association between amylase gene $\mathrm{CN}$ and adiposity traits in a large Middle Eastern population. Importantly, we leverage rich biobank data to demonstrate that the strength of this association varies with ethnicity, and may be influenced by population-specific behaviors that also contribute to adiposity traits.

npj Genomic Medicine (2021)6:8; https://doi.org/10.1038/s41525-021-00170-3

\section{INTRODUCTION}

Salivary (AMY1) and pancreatic (AMY2A) amylase enzymes are responsible for starch digestion, which begins in the oral cavity and continues in the small intestine. The AMY1 and AMY2A genes show extensive copy number $(\mathrm{CN})$ variability in humans, with a reported number of gene copies ranging from 2 to 18 for $A M Y 1$, and from 0 to 8 for $A M Y 2 A^{1-4}$. It has been shown that $C N$ distribution at the salivary amylase gene is significantly variable between populations, with the number of copies of $A M Y 1$ reflecting a biological adaptation to traditionally high-starch or low-starch diets throughout evolution ${ }^{5}$.

In 2014, we first reported an association between reduced $A M Y 1$ $\mathrm{CN}$ and increased body mass index (BMI) and obesity risk using 6200 individuals of European and Asian ancestries ${ }^{6}$, although subsequent studies attempting to replicate this association yielded conflicting results. For instance, association with $A M Y 1$ $\mathrm{CN}$ was not replicated in 4000 individuals of European ancestry, including people selected for being at the extremes of the BMI distribution $^{3}$, as well as in a case-control study of 932 Chinese and 145 Malay samples ${ }^{7}$, and in 1400 participants from the UK 1958 Birth Cohort ${ }^{8}$. On the other hand, studies specifically analyzing obesity in children and young adults supported the association of BMI with amylase gene copy number in French ${ }^{9}$, Mexican ${ }^{10}$, and Italian children ${ }^{11}$, as well as in females with early-onset obesity from Finland ${ }^{12}$.

These studies highlight the complexity of studying an endpoint which has both genetic and environmental components, and suggest that differences in ethnicity, environment and food preferences may further influence the manifestation of this complex phenotype in the setting of genetic susceptibility.
Indeed, previous works exploring the relationship between $A M Y 1 \mathrm{CN}$ and diet revealed a significant effect of the interaction between AMY1 CN and starch intake on BMI in 4800 nondiabetic adults from Sweden ${ }^{13}$, and greater weight and central adiposity loss following randomized low-calorie diet interventions among carriers of the allele rs11185098-A (a proxy of higher AMY1 CN and activity $^{3}$ ), compared to noncarriers, among 692 Europeans from The POUNDS Lost Trial ${ }^{14}$. Taken together, these studies suggest that environmental factors, and particularly dietary choices, may play a role in modulating the observed association between AMY1 $\mathrm{CN}$ and adiposity.

Here, we report a large-scale association study between $\mathrm{CN}$ at the amylase genes $(A M Y 1$ and $A M Y 2 A)$ and adiposity traits in a large Middle Eastern cohort. Specifically, we combined CN inference from high-coverage $(30 \times)$ whole-genome sequencing (WGS) with phenotypic traits related to adiposity traits and behaviors, collected for almost 3000 subjects as part of the Qatar Biobank $^{15}$. Our findings help explain trans-ethnic differences in the effect of amylase $\mathrm{CN}$ on adiposity and introduce a role for subpopulation-specific traditional dietary and lifestyle choices in determining the strength of association between amylase and adiposity in global populations.

\section{RESULTS}

Distribution of adiposity traits in the Qatari population

The Qatar Biobank (QBB) collected a wide range of traits, biochemical measurements, and lifestyle questionnaires from adult Qatari nationals and long-term residents ${ }^{15}$. While many of these traits will be available for future studies, we focused this first

\footnotetext{
${ }^{1}$ Department of Twin Research and Genetics Epidemiology, King's College London, London, UK. ${ }^{2}$ Department of Human Genetics, Sidra Medicine, Doha, Qatar. ${ }^{3}$ Department of Genetic Medicine, Weill-Cornell Medical College, Doha, Qatar. ${ }^{4}$ College of Health and Life Science, Hamad Bin Khalifa University, Doha, Qatar. ${ }^{5}$ These authors contributed equally: Niccolo' Rossi, Elbay Aliyev. ${ }^{6}$ These authors jointly supervised this work: Khalid A. Fakhro, Mario Falchi. ${ }^{\circledR}$ email: kfakhro@sidra.org
} 
analysis of QBB data on adiposity traits, specifically: BMI, total fat percentage, and trunk fat percentage.

Among the 2935 individuals for whom genomic data was available as part of the Qatar Genome Programme (QGP) pilot phase, 1464 were females and 1471 males. Measures of BMI, total and trunk fat percentages were normally distributed with means (SD) of 28.9 (5.7), 32.7 (9.7) and 32.8 (9.1), respectively (Table 1, Fig. 1). Mean age was not statistically different between sex groups (Wilcoxon's $P>0.05$ ). Conversely, all the adiposity traits under study were significantly higher in female than in male subjects (Table 1, Fig. 1; Wilcoxon's $P<5.08 \times 10^{-5}$ ), with the strongest difference being observed for total fat percentage (Wilcoxon's $P=1.54 \times 10^{-295}$ ).

\section{Range of $A M Y 1$ and $A M Y 2 A$ copy number variability in the Qatari population}

We first aimed to estimate the range of discrete $\mathrm{CN}$ variation at human salivary $(A M Y 1)$ and pancreatic $(A M Y 2 A)$ amylase genes in the Qatari population by coverage analysis of WGS data from 2935 individuals from the QBB (see "Methods" section).

Following post-mapping quality control of reads from wholegenome sequencing data (see "Methods" section), we found that

Table 1. Adiposity traits of 2935 QBB individuals sequenced as part of the QGP pilot phase.

\begin{tabular}{|lllll}
\hline & All & Females & Males & $P$ \\
\hline$N$ & 2935 & 1471 & 1464 & - \\
Age (years) & $39.11 \pm 12.03$ & $39.50 \pm 12.62$ & $38.72 \pm 11.42$ & $>0.05$ \\
& {$[29.00-48.00]$} & {$[29.00-49.00]$} & {$[30.00-46.00]$} & \\
BMI (kg/m $\left.{ }^{2}\right)$ & $28.86 \pm 5.71$ & $29.33 \pm 6.28$ & $28.36 \pm 5.00$ & $5.08 \times 10^{-5}$ \\
& {$[24.90-32.40]$} & {$[24.90-33.40]$} & {$[24.90-31.30]$} & \\
Total fat (\%) & $32.69 \pm 9.72$ & $39.15 \pm 7.56$ & $25.96 \pm 6.68$ & $1.54 \times 10^{-295}$ \\
& {$[25.70-40.20]$} & {$[34.8-44.5]$} & {$[22.00-30.33]$} & \\
Trunk & $32.82 \pm 9.07$ & $36.70 \pm 8.93$ & $28.77 \pm 7.27$ & $3.71 \times 10^{-138}$ \\
fat (\%) & {$[27.50-38.90]$} & {$[31.90-43.00]$} & {$[24.67-33.70]$} & \\
\hline
\end{tabular}

Reported here are means, standard deviations, 1st-3rd interquartile ranges (in brackets), and statistical differences between sex groups (Wilcoxon's test $p$-values are reported). the percentage of correctly aligned reads ranged from 90 to $97 \%$, for $A M Y 1$ (mean $=95.2 \%$, median $=95.3 \%$, interquartile range $[\mathrm{IQR}]=94.7-95.8 \%$ ), and from 90 to $99 \%$, for $A M Y 2 A$ (mean = 94.5\%, median $=94.7 \%$, IQR = 94.2-95.0\%; Supplementary Fig. 1). In addition, the average per-individual percentage of reads with mapping quality score $>20$ and uniquely mapping to both $A M Y 1$ and $A M Y 2 A$ was $99.99 \%$, corresponding to a probability of inaccurate mapping of at most 0.01 (Supplementary Fig. 1).

In the Qatari population, $A M Y 1$ gene copy number ranges from 2 to $22, A M Y 2 A$ from 0 to 5 , and $A M Y 2 B$ from 1 to 5 copies. Consistent with previous reports ${ }^{1,3,6,16}$, the number of copies of $A M Y 1$ and $A M Y 2 A$ were significantly correlated to each other (polychoric correlation estimate $\rho=0.28, \quad P<2.22 \times 10^{-308}$; Fig. 2b), with $72 \%$ of the Qatari population carrying an even diploid number of $A M Y 1$ genes and two copies of $A M Y 2 A$.

The number of copies of $A M Y 2 A$ and $A M Y 2 B$ showed high correlation (Pearson's $\rho=0.81, P<2.22 \times 10^{-308}$ ), corresponding to $90 \%$ of QBB samples carrying the same number of copies of these two genes. In $88 \%$ of the discordant CN calls at $A M Y 2 A$ and $A M Y 2 B$, $A M Y 2 A$ arbored $C N$ variations, whereas $A M Y 2 B$ was copy-invariable $(\mathrm{CN}=2)$. This is consistent with previous studies reporting $95 \%$ of haploid similarity between $A M Y 2 A$ and $A M Y 2 B C N^{2,3}$, and substantial higher $C N$ variation ( $24 \%$ vs. $10 \%$, respectively) at $A M Y 2 A$ compared to $A M Y 2 B^{2}$. Due to the extensive overlap between $A M Y 2 A$ and $A M Y 2 B C N$, and the higher variability observed at the $A M Y 2 A$ locus compared to $A M Y 2 B$, when these were $C N$-discordant, $A M Y 2 B$ was excluded from subsequent analyses.

We further investigated the presence of damaging loss-offunction (LoF) variants within the coding region of $A M Y 1$ and $A M Y 2 A$ and which may affect the number of active copies of the genes and therefore skew the results (see "Methods" section); however we found no variants with putative disruptive effects (i.e., stop gained, frameshift, splice variants or in-frame deletions). Indeed, the frequency of LoF variants in $A M Y 1$ and $A M Y 2 A$ is reported to be rare $(M A F<1 \%)$ in the general population based on the Genome Aggregation Database (gnomAD) ${ }^{17}$.

\section{Validation of amylase copy number estimates}

We estimated $A M Y 1$ and $A M Y 2 A C N$ on 40 samples using digital droplet PCR (ddPCR; Methods), identifying only six discrepancies with respect to the CNs estimated with the Carpenter et al.'s approach ${ }^{1}$ used here (five for $A M Y 1$ calls and one for $A M Y 2 A$ calls, Supplementary Table 1), therefore confirming a very high accuracy $(74 / 80=92.5 \%)$ of our calls across both loci. Notably, four out of five instances of discrepancy at $A M Y 1$ deviated from the ddPCR
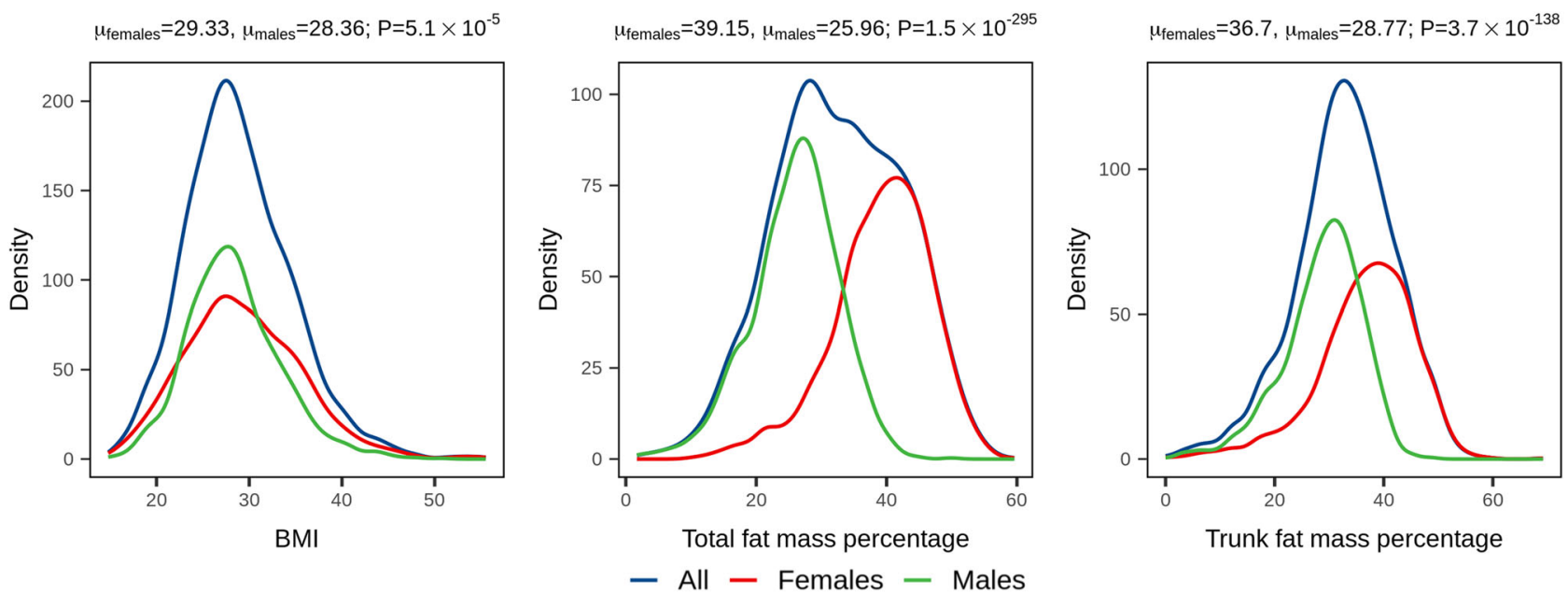

Fig. 1 Distribution of adiposity traits. Density plots showing BMI, total and trunk fat percentages distribution in the Qatari population $(N=$ 1471 and 1464 females and males, respectively). The mean trait value ( $\mu$ ), stratified by sex, was evaluated by means of the Wilcoxon's test, and $p$-values are reported above each panel. 
a
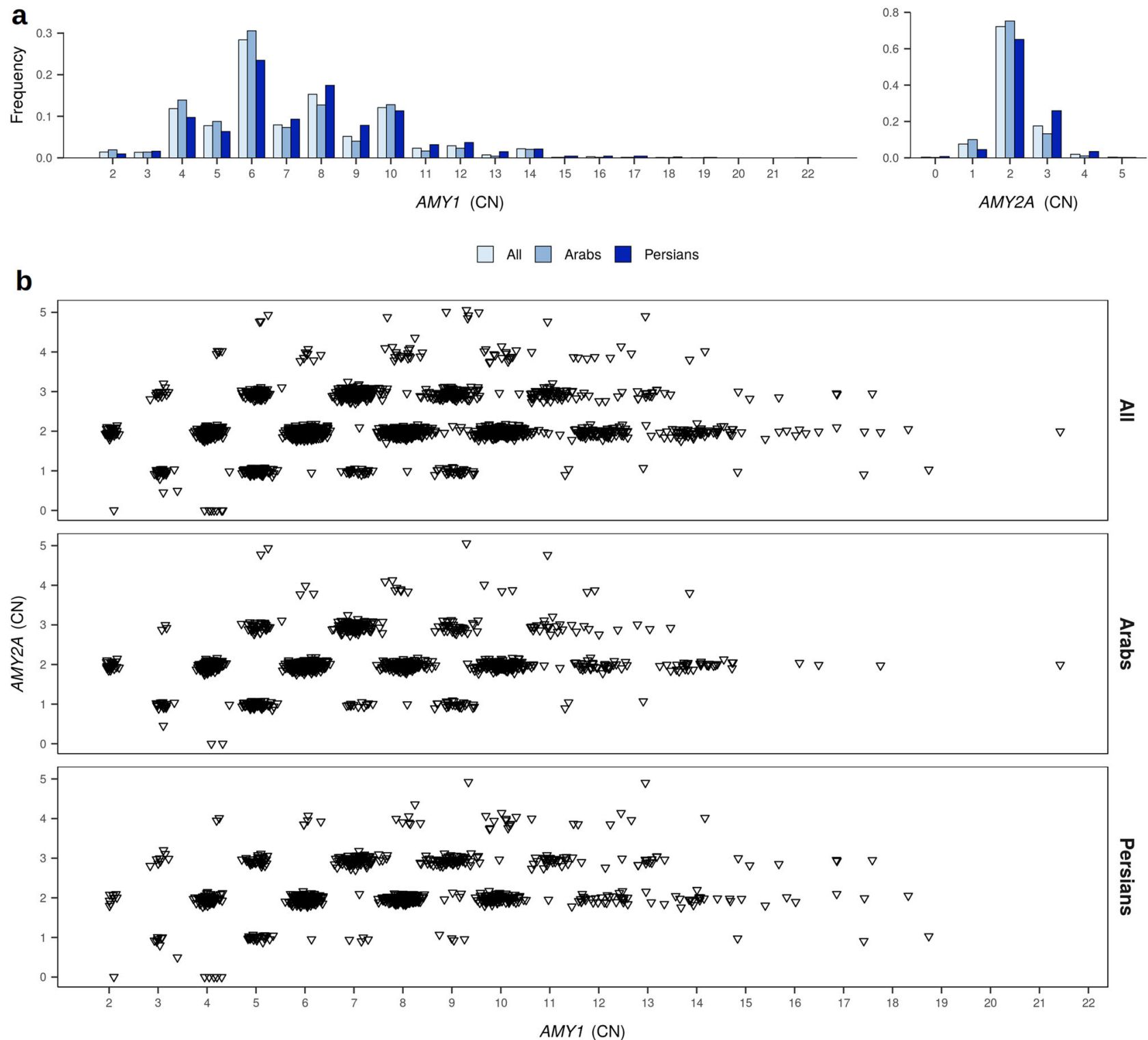

Fig. $2 A M Y 1$ and $A M Y 2 A$ variation in the Qatari population. a Histograms showing the distribution of estimated copy numbers of $A M Y 1$ and $A M Y 2 A$, obtained from coverage analysis of whole-genome sequencing data in all the Qatari population $(N=2935$, white), and Arabs $(N=$ 1518 , light blue) and Persians ( $N=948$, dark blue) from Qatar. b In the Qatari population, $A M Y 2 A$ and $A M Y 1$ show parity: when $A M Y 2 A$ copy number is odd, so is $A M Y 1$ copy number.

results for a single copy, with consequent deviation from shared odd/even parity between $A M Y 1$ and $A M Y 2 A C N$. Such a deviation is reported to have a low frequency in the general population ${ }^{3}$, and was avoided in our analysis, where conditional rounding was applied.

We used $A M Y C N E^{18}$ to further validate the $A M Y 1 C N$ inferred using the approach described by Carpenter et al. ${ }^{1}$ and observed a strong agreement between the two approaches (Pearson's $\rho=$ 0.98; $P<2.22 \times 10^{-308} ; N=2935$, Supplementary Fig. 2).

\section{Association of amylase genes CNs with adiposity in the Qatari population}

Anthropometric measurements of adiposity (BMI, total fat percentage, and trunk fat percentage) were tested for association with $A M Y 1$ and $A M Y 2 A C N$ in 2935 individuals from Qatar using PopPAnTe ${ }^{19}$, including sex, age at the sample collection, and the first 10 PCs from genome-wide data as covariates (see "Methods" section). At a Bonferroni-adjusted significance threshold of $8.33 \times$
$10^{-3}$ (see "Methods" section), $A M Y 1$ appeared significantly associated with trunk fat percentage $(\beta=-0.02, \mathrm{SE}=0.01 ; P=$ $7.50 \times 10^{-3}$; Table 2). Conversely, no significant association was observed for AMY2A ( $P>0.05$; Table 2).

\section{Qatari genetic subpopulations}

Since the Qatari population includes subjects of different ancestries, we further sought to distinguish them within our sample and assess their contribution to the observed association between $A M Y 1$ and adiposity. To identify the underlying genetic ancestries, we performed principal component analysis (PCA) using ancestry informative markers ${ }^{20,21}$ and identified individuals clustering with three major Qatari subpopulations (namely, Bedouin Arabs, Persians and East Africans; see "Methods" section, Fig. 3). We excluded from further analyses East Africans, because of their small sample size $(N=63)$, along with admixed individuals $(N=406)$ which could not be assigned to a majority ethnic 
Table 2. Associations between amylase copy number and adiposity traits in the Qatari population.

\begin{tabular}{llllll}
\hline Gene & Trait & $N$ & $\beta$ & SE & $P$ \\
\hline AMY1 & BMI $\left(\mathrm{kg} / \mathrm{m}^{2}\right)$ & 2908 & -0.02 & 0.01 & $3.26 \times 10^{-2}$ \\
AMY1 & Total fat $(\%)$ & 2740 & -0.01 & 0.01 & $1.10 \times 10^{-2}$ \\
$A M Y 1$ & Trunk fat (\%) & 2726 & -0.02 & 0.01 & $7.50 \times 10^{-3}$ \\
$A M Y 2 A$ & BMI $\left(\mathrm{kg} / \mathrm{m}^{2}\right)$ & 2897 & -0.07 & 0.03 & $2.92 \times 10^{-2}$ \\
$A M Y 2 A$ & Total fat $(\%)$ & 2729 & -0.04 & 0.02 & 0.108 \\
AMY2A & Trunk fat (\%) & 2715 & -0.06 & 0.03 & 0.050 \\
\hline
\end{tabular}

Association testing between amylase genes $\mathrm{CN}$ and inverse normalized adiposity traits was performed in 2935 Qataris using PopPAnTe, which takes into account individuals' kinship, age, sex and the first 10 PCs from genome-wide data as covariates. The table reports the number of samples included in the tested datasets $(\mathrm{N})$, the effect sizes $(\beta)$ with their standard errors (SE), and the association $p$-values $(P)$.

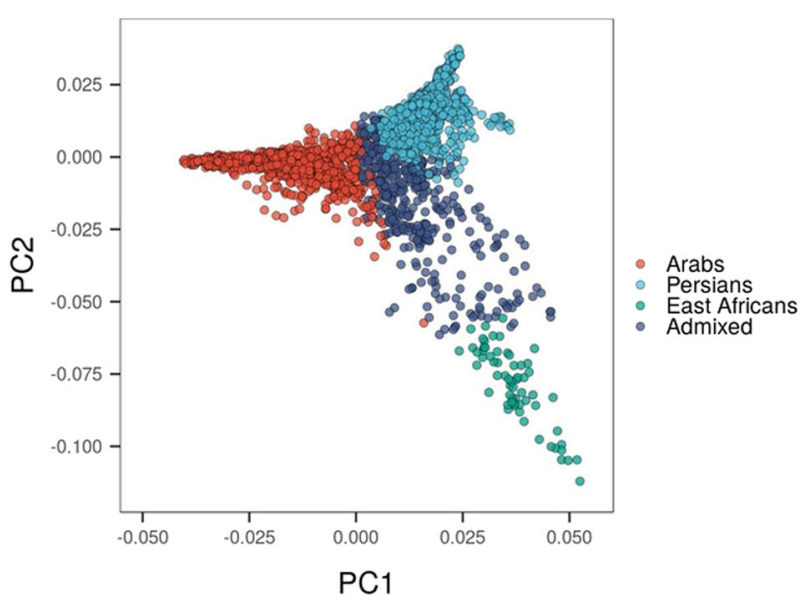

Fig. 3 Principal component analysis. Scatterplot of the first two principal components assessed on ancestry-informative SNPs of 2,935 individuals from Qatar (see "Methods" section). Individual data points are color-coded according to previously described major ethnic ancestry: Arabs (red), Persians (light blue), and East Africans (green). Admixed individuals (purple) are those whose majority ancestry cannot be assigned to one group.

ancestry. Persians $(N=948)$ and Arabs $(N=1518)$ were thus selected for subsequent study.

To avoid further demographic confounders, we investigated but did not find differences between the two groups in age ( $\mu_{\text {Persians }}$ $=40,3 ; \mu_{\text {Arabs }}=39.1$; Wilcoxon's $\left.P=0.02\right)$ or sex distribution $\left(x^{2} P\right.$ $>0.05$ ). Despite the similar make-up of the two groups, Qataris with Persian ancestry appeared to have an overall healthier adiposity profile than those with Arab ancestry, as measured by BMI $\left(\mu_{\text {Arabs }}=29.25 ; \mu_{\text {Persians }}=27.84 ;\right.$ Wilcoxon's $\left.P=1.34 \times 10^{-8}\right)$, total fat $\left(\mu_{\text {Arabs }}=33.68 ; \mu_{\text {Persians }}=31.00 ; P=2.1 \times 10^{-10}\right)$ and trunk fat percentages $\left(\mu_{\text {Arabs }}=33.89 ; \mu_{\text {Persians }}=31.02 ; P=1.3 \times 10^{-13}\right)$, all of which were significantly lower in Persians as compared to Arabs (Fig. 4). Furthermore, consistent with the distribution from the overall group, within each ethnic group total and trunk fat percentages were remarkably higher in females than males, though this difference was less pronounced in Persians (Wilcoxon's $P_{\text {Arabs }}<4.90 \times 10^{-86} ; P_{\text {Persians }}<9.18 \times 10^{-33}$; Fig. 4). Conversely, females were observed to have significantly higher BMI than males ( $P=9.90 \times 10^{-7}$; Fig. 4) only among Arabs.

\section{$A M Y 1$ and $A M Y 2 A$ CNs are higher in Qataris with Persian ancestry}

$A M Y 1$ and $A M Y 2 A C N$ s showed significant correlations in both Arabs and Persians (polychoric correlation estimate $\rho_{\text {Arabs }}=0.27$, $P=4.57 \times 10^{-315} ; \rho_{\text {Persians }}=0.29, P=1.91 \times 10^{-201} ;$ Fig. $2 \mathrm{~b}$ ), with 75 and $65 \%$ of the Arab and Persian subpopulation, respectively, carrying an even number of $A M Y 1$ genes and two copies of AMY2A.

We observed significant differences in $\mathrm{CN}$ distribution at $A M Y 1$ and $A M Y 2 A$ between Persians and Arabs $\left(X^{2}\right.$ test $P=7.23 \times 10^{-12}$ and $P=9.15 \times 10^{-21}$, respectively), with Persian subjects appearing to have alleles with a higher number of copies of $A M Y 1$ ( $\mu_{\text {Arabs }}$ $=6.92$ copies; $\mu_{\text {Persians }}=7.60$ copies; Wilcoxon's test $P=1.32 \times$ $10^{-10}$; Fig. $\left.2 \mathrm{a}\right)$, as well as $A M Y 2 A\left(\mu_{\text {Arabs }}=2.06\right.$; $\mu_{\text {Persians }}=2.27$; Wilcoxon's test $P=7.49 \times 10^{-21}$; Fig. 2a).

Differences in $A M Y 1$ and $A M Y 2 A$ CNs distribution between Arabs and Persian were supported by differences in estimated haplotypes frequencies at both loci (Supplementary Methods; Supplementary Results, Supplementary Fig. 3).

\section{Genetic ancestry at the $A M Y 1$ locus}

To rule out the possibility that the amylase locus may not reflect the overall genetic ancestry assigned to each individual based on genome-wide markers, we performed a further PCA using SNPs and indels located within a 5-MB window surrounding AMY1 (see "Methods" section). Our analysis confirmed that individuals of Arab and Persian ancestry were still well separated in this novel principal component space (Supplementary Fig. 4), while also highlighting the presence of a small subset of outliers $\left(N_{\text {Arabs }}=75\right.$, $N_{\text {Persians }}=34$ ), likely reflecting finer ancestry substructures within these main ancestry groups. Notably, we investigated whether the exclusion of these individuals would influence the association signals identified in our primary analysis, and found negligible differences (see "Methods" section, Supplementary Table 2).

\section{Association between $A M Y 1 \mathrm{CN}$ and adiposity traits in Arabs and Persians}

Total fat percentage, BMI, and trunk fat percentage were tested for association with AMY1 CN in 948 Persians and 1518 Arabs from Qatar using PopPAnTe ${ }^{19}$. Sex and age at sample collection were included as covariates, as well as the first 10 PCs from genomewide data (see "Methods" section).

At a Bonferroni-adjusted significance threshold of $8.33 \times 10^{-3}$ (see "Methods" section), we observed negative associations between $A M Y 1 \mathrm{CNs}$ and both total and trunk fat percentages in Arabs $\left(P<4.60 \times 10^{-3}\right.$; Table 3, Fig. 5). In addition, lower $A M Y 1 \mathrm{CNs}$ was nominally associated with increased BMl in Arabs $(P=8.40 \times$ $\left.10^{-3}\right)$. Conversely, no significant association $(P>0.05)$ was observed in Persians; however, the direction of association of $A M Y 1$ with adiposity was concordant between ethnic groups (Table 3).

In addition, case-control analysis of 593 obese $\left(\mathrm{BMI} \geq 30 \mathrm{~kg} / \mathrm{m}^{2}\right.$ ) and 357 normal weight $\left(\mathrm{BMl}<25 \mathrm{~kg} / \mathrm{m}^{2}\right)$ Arab individuals from the same dataset highlighted that higher $A M Y 1 C N$ was indeed inversely associated with obesity risk $(\mathrm{OR}=0.93,95 \% \mathrm{Cl}=$ [0.88-0.98]; $P=0.01$ ).

\section{Contribution of population specific behaviors to $A M Y 1$ association with adiposity}

Both $A M Y 1$ copy number variability and its contribution to adiposity are thought to arise as a product of the adaptive interaction between $A M Y 1$ and environmental exposure to starch throughout evolution ${ }^{5}$. As a consequence, it is prudent to consider that diverse ancestral environments and differences in lifestyle choices (e.g., dietary behavior) may have impacted AMY1 CNVs association with adiposity in Persian and Arab Qataris. 

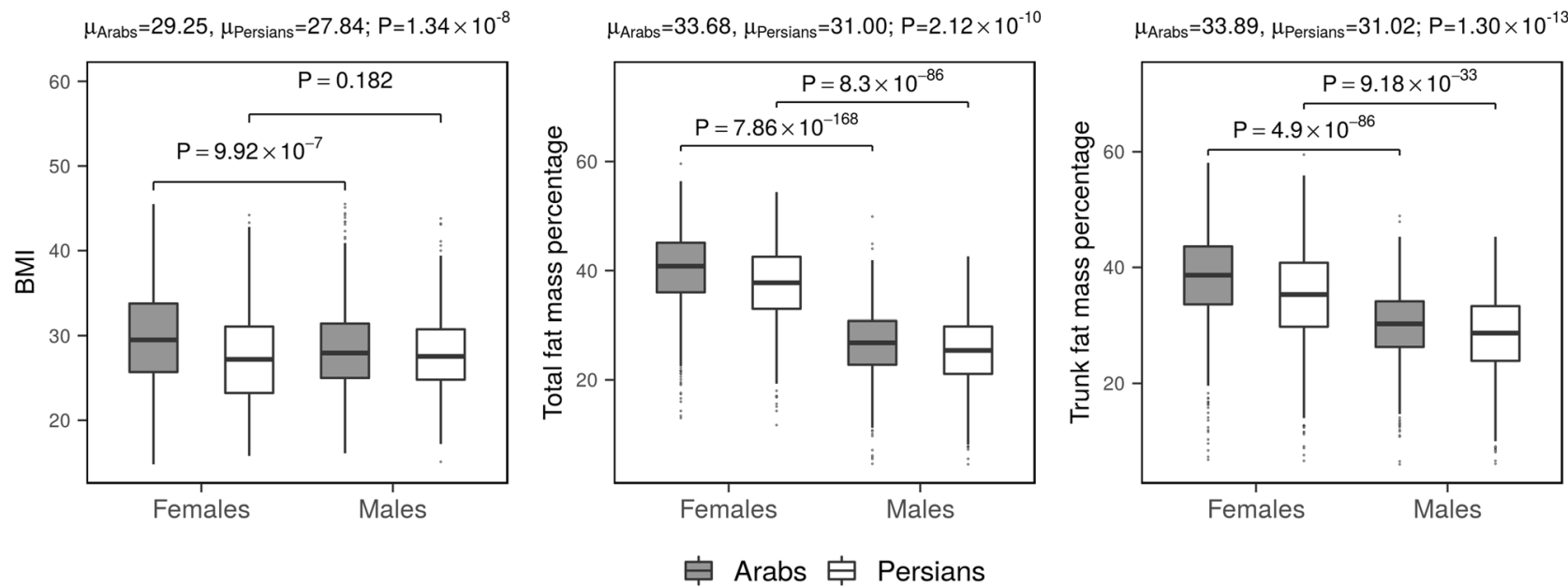

Fig. 4 Distribution of adiposity traits in Arabs and Persians from Qatar. Boxplots showing BMl, total and trunk fat percentages distribution in Arabs $(N=1518$, gray) and Persians $(N=948$, blue). The center line of the boxplots indicates the median, limits of the box indicate the 25 th and 75 th percentile. The whiskers represent either 1.5 times the interquartile range (IQR) or the maximum/minimum data point if they are within 1.5 times the IQR. The mean trait value $(\mu)$ stratified by ethnic group and the $p$-values, evaluated by means of the Wilcoxon's test, are reported above each panel. Sex-stratified $p$-value within each ethnic group are reported within each panel.

Table 3. Associations between $A M Y 1$ copy number and adiposity traits in Persians and Arabs from Qatar.

\begin{tabular}{llrlll}
\hline Ancestry & Trait & $N$ & \multicolumn{1}{l}{$\beta$} & \multicolumn{1}{l}{ SE } & $P$ \\
\hline Arabs & BMI $\left(\mathrm{kg} / \mathrm{m}^{2}\right)$ & 1505 & -0.03 & 0.01 & $4.60 \times 10^{-3}$ \\
Arabs & Total fat (\%) & 1432 & -0.02 & 0.01 & $8.40 \times 10^{-3}$ \\
Arabs & Trunk fat (\%) & 1425 & -0.03 & 0.01 & $4.40 \times 10^{-3}$ \\
Persians & BMI $\left(\mathrm{kg} / \mathrm{m}^{2}\right)$ & 944 & 0.00 & 0.01 & 0.804 \\
Persians & Total fat (\%) & 879 & -0.01 & 0.01 & 0.300 \\
Persians & Trunk fat (\%) & 874 & -0.01 & 0.01 & 0.320
\end{tabular}

Association testing between $A M Y 1 \mathrm{CN}$ and inverse normalized adiposity traits was performed in 1518 Arabs and 948 Persians from Qatar using PopPAnTe, which takes into account individuals' kinship, age, sex and the first 10 PCs from genome-wide data as covariates. The table reports the number of samples included in the tested datasets $(N)$, the effect sizes $(\beta)$ with their standard errors (SE), and the association $p$-values $(P)$.

Among study subjects, 1805 individuals (1148 Arabs and 657 Persians) were overweight or obese $\left(B M I \geq 25 \mathrm{~kg} / \mathrm{m}^{2}\right)$. Notably, in reviewing the QBB intake questionnaires, we found that 1013 of these declared to have modified their diet during the year of data collection because of excess weight and its related comorbidities (e.g., high cholesterol, hypertension, and diabetes). Furthermore, we observed by multiple logistic regression (see "Methods" section) a significant difference between Arabs and Persians in the tendency towards dietary self-restraint following weight gain, with 54 and $60 \%$ of overweight or obese Arabs and Persians restraining their diet, respectively $\left(P=4.29 \times 10^{-5}\right)$. In the same analysis, sex also appeared to be a significant $\left(P=1.36 \times 10^{-6}\right)$ determinant of dietary behaviors in overweight or obese subjects from Qatar, with $60 \%$ of overweight females (58 and 65\% in Arabs and Persians, respectively) displaying dietary restraint, as compared to only $52 \%$ of overweight males (49 and $56 \%$ in Arabs and Persians, respectively). Interestingly, higher $A M Y 1$ copy number was negatively associated with diet restraint in overweight subjects (OR per estimated unit copy increase $=0.94,95 \% \mathrm{Cl}=$ $\left.0.91-0.98 ; P=3.22 \times 10^{-3}\right)$.

\section{Effect of ethnicity on the association between $A M Y 1$ and adiposity traits and on dietary restraint}

It could be hypothesized that if $A M Y 1 \mathrm{CN}$ segregates with ancestry, such that increased Arab ancestry corresponds to decreased $A M Y 1 \mathrm{CN}$, and at the same time genetic factors other than $A M Y 1$ increase the risk of adiposity in Arabs, such that increased Arab ancestry corresponds to increased adiposity, then these two independent events would generate a spurious association between AMY1 CN and adiposity traits. To investigate this further, we explored the relationship between varying degrees of ancestry, as defined using the first principal component (PC1) values (see "Methods" section) and both AMY1 $\mathrm{CN}$ and adiposity traits.

After accounting for age and sex, adiposity traits showed significant negative associations with $\mathrm{PC} 1$ among both individuals of Arab ancestry $(\beta=-4.66, \mathrm{SE}=2.47, P=0.06 ; \beta=-4.85, \mathrm{SE}=$ $1.79, P=6.88 \times 10^{-3} ; \beta=-6.31, \mathrm{SE}=2.12, P=3.02 \times 10^{-3}$, for $\mathrm{BMI}$, total and trunk fat and total fat percentages, respectively) and Persian ancestry $\left(\beta=-18.53, \mathrm{SE}=6.18, P=2.77 \times 10^{-3} ; \quad \beta=\right.$ -14.27, $\mathrm{SE}=4.75, P=2.73 \times 10^{-3} ; \beta=-18.90, \mathrm{SE}=5.79, P=$ $1.13 \times 10^{-3}$, for BMl, total and trunk fat and total fat percentages, respectively; Supplementary Fig. 5). Conversely, $A M Y 1 \mathrm{CN}$ did not associate with PC1 in Persians $(P=0.23)$, and only weakly in Arabs $(\beta=-13.57, \mathrm{SE}=6.57, P=0.04$; Supplementary Fig. 5$)$, where an increased Arab ancestry actually associated with higher copy number, i.e., in the opposite direction compared to our original association results. Therefore, our main findings do not appear to be biased by potential co-segregation of adiposity and AMY1 CN with ancestry.

Interestingly, while no difference in the association between $A M Y 1$ and adiposity was observed between subgroups of subjects characterized by varying degrees of Persian ancestry, the higher the Arab ancestry, the stronger the association with all adiposity traits (see "Methods" section, Supplementary Fig. 6). To explore what could drive this behavior, we evaluated the heritability of the three adiposity traits at varying degrees of ancestry (see "Methods" section). While, in general, the heritability of all adiposity traits was higher among Persians (42-44\%) compared to Arabs (19-23\%), Arabs (but not Persians) showed decreased heritability of these traits with increased ancestry (Supplementary Fig. 6). This was not driven by a decreased number or strength of pairwise kinship between subjects with strongest Arab ancestry, 
$\rho_{=}=0.068 ; P=8.5 \times 10^{-3}$

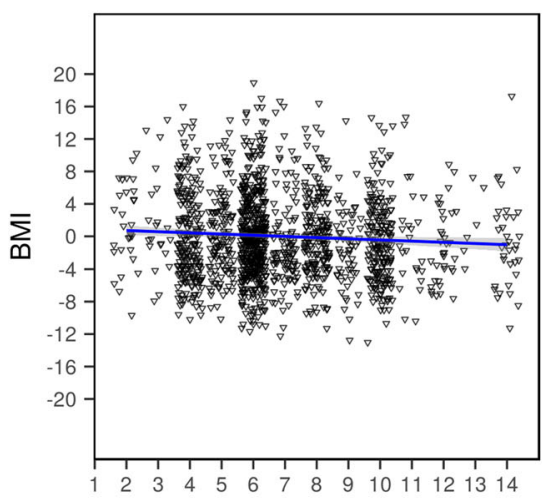

AMY1 (CN) $\rho=-0.069 ; P=9.1 \times 10^{-3}$

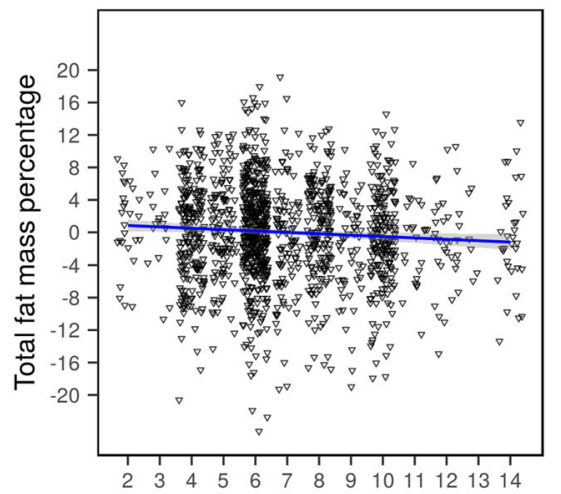

AMY1 (CN) $\rho=0.071 ; P=7.5 \times 10^{-3}$

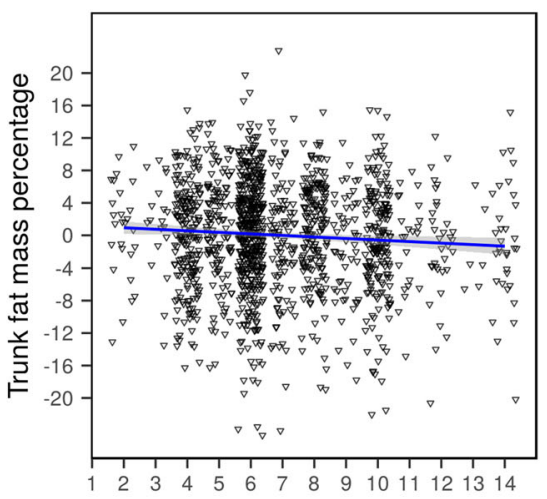

AMY1 (CN)

Fig. 5 AMY1 CN association with adiposity in Arabs from Qatar. The scatter plots depict the correlation between salivary amylase copy number ( $x$-axis) and sex-corrected and age-corrected measures of adiposity ( $y$-axis). Pearson's correlation coefficients $(\rho)$ and $p$-values are reported above each panel.

which might have deflated the heritability estimate. Instead we hypothesized that this could have been caused by ethnic-specific behavioral or environmental factors. We further assessed the association between PC1 and dietary behavior, finding a significant association between the degree of Arab ancestry and decreased tendency towards dietary restraint $\left(P=1.26 \times 10^{-5}\right.$; Supplementary Fig. 7), while dietary restraint behavior did not change between subgroups of subjects characterized by varying degrees of Persian ancestry $(P>0.05)$. Consistent with this finding, we observed a positive association between $A M Y 1 \mathrm{CN}$ and dietary restraint with increased Arab ancestry adjusting for age, sex and BMI, compared to Persians subjects (Supplementary Fig. 8).

\section{DISCUSSION}

Elucidating the relationship between genetic architecture and obesity-related traits is of fundamental scientific and public-health concern, primarily due to the chronic comorbidities associated with obesity and the increased risk of premature mortality across various human populations ${ }^{22}$. The national STEPwise survey conducted in Qatar in 2012 reported an obesity epidemic (>70\% of the adult population had a BMI $\geq 25 \mathrm{~kg} / \mathrm{m}^{2}$ ), accompanied by an alarming rate of comorbidities in the affected population, such as hypertension (32.9\% of respondents aged 18-64) and type II diabetes $(17.6 \% \text { of men and } 15.9 \% \text { of women })^{23}$.

While previous works have investigated the association of SNPs, epigenetic markers and obesity in the Gulf Arab world ${ }^{24-27}$, no studies to date have uncovered copy number variants influencing adiposity in this population.

Here, we discretely genotyped the range of copy number variation at $A M Y 1$ and $A M Y 2 A$ genes through coverage analysis of $30 \times$ whole genome sequencing data in 2935 individuals from Qatar, and showed that (1) copy number at both genes has similar distribution and correlation to previous reports ${ }^{1,3,6,16}$, and (2) $A M Y 1 C N$ is negatively associated with trunk fat percentage.

Putative mechanisms by which $A M Y 1$ may be linked to obesity involves its role in modulating starch intake, sweet-taste perception, and carbohydrate digestion ${ }^{28,29}$ and gut microbiome composition ${ }^{30,31}$. It has been reported that subjects expressing lower $A M Y 1$ levels show higher blood glucose and delayed insulin response following starch ingestion ${ }^{28}$, suggesting that carriers of lower AMY1 CN may be at higher risk of developing insulin resistance if chronically relaying on starch-rich diets. In addition, increased ketonuria is associated with low serum amylase ${ }^{32}$ which further supports the hypothesis that individuals with lower AMY1 $\mathrm{CN}$ may not be fully adapted to a carbohydrate-based diet.
Indeed, a combined mass-spec and NMR metabolomics study of homogeneous and age-matched normal weight French adult females highlighted increased reliance on lipid vs glucose metabolism for energy production in low CN carriers at $A M Y 1^{33}$.

Studies exploring how the human gut microbiome responds to $A M Y 1 \mathrm{CN}$ variation yielded conflicting results. For instance, it has been shown that gut microbiome of individuals carrying high $A M Y 1$ copies is enriched in microbes involved in complex polysaccharide degradation belonging to the Prevotella genus ${ }^{30}$, and that high Prevotella/Bacteroides ratio favors weight loss in response to dietary interventions ${ }^{34,35}$. Conversely, Poole and colleagues reported adiposity gain in germ-free mice following fecal microbiota transplantation from carriers of high $A M Y 1 \mathrm{CN}^{31}$.

Whereas the hypothesis that high AMY1 CN promotes obesity by modulating starch metabolism has still to be fully proven, it is known that $A M Y 1 \mathrm{CN}$ has followed different evolutionary trajectories between populations, as a function of the variability in starch content across traditional diets ${ }^{5}$. Given the ethnic heterogeneity of Qataris from $\mathrm{QBB}^{20,21}$, we sought to dissect possible ancestryspecific contributions to $A M Y 1 C N$ association with adiposity in Qatar. Interestingly, we found that Arabs from Qatar (and females in particular) have, on average, unhealthier adiposity profiles, and significantly lower $A M Y 1$ and $A M Y 2 A C N s\left(P_{A M Y 1}=1.32 \times 10^{-10}\right.$ and $P_{A M Y 2 A}=7.49 \times 10^{-21}$, respectively) compared to Qataris of Persian ancestry. Higher $A M Y 1$ and $A M Y 2 A C N$ in Persians compared to Arabs from Qatar are consistent with differences in ancestral diets across the two ethnic groups. Indeed, whereas the traditional diet of populations with a history of nomadic pastoralism (e.g., Bedouin Arabs) relies extensively on proteinaceous resources and simple saccharides, starchy food resources traditionally comprise a substantial portion of the diet in traditional agricultural societies, such as Persians $5^{5}$. Higher ancestral $A M Y 1$ and $A M Y 2 A C N$ in Persians from Qatar compared to Arab may have resulted in increased resilience, as supported by their more favorable adiposity profile, to dietary changes introduced by the rapid growth and occidentalization of Qatar brought about by newfound oil-wealth, which paralleled the increase of obesity prevalence ${ }^{36}$.

Despite showing similar negative trends, the association between $A M Y 1 \mathrm{CNs}$ and measures of adiposity reached statistical significance only in Arabs, possibly due to lack of statistical power when testing Persians, given the smaller sample size or, indeed, population-specific effects.

We leveraged responses from the QBB on self-reported dietary restraint and observed a significant difference between Arabs and Persians in their tendency towards dietary restraint following weight gain $\left(P=4.29 \times 10^{-5}\right)$, with $60 \%$ of overweight or obese 
Persians restraining their diet compared to $54 \%$ of their Arab counterpart. In addition, we showed that increased Arab ancestry is associated with both decreased tendency towards dietary restraint following weight gain and increased association strength between AMY1 CN and dietary behavior, thus providing further evidence of the complexity of the association between AMY1 and adiposity, as it can be affected by population-specific environmental factors. This is supported by previous studies reporting a significant effect of the interaction between $A M Y 1 \mathrm{CN}$ and dietary starch intake on $\mathrm{BMI}^{37}$, and an association between $A M Y 1$ and $\mathrm{BMI}$ in overweight individuals on dietary intervention ${ }^{14}$. Unfortunately, food frequency questionnaires were not available to us for this cohort, and therefore we could not assess the contribution of dietary starch intake to the association between $A M Y 1 \mathrm{CN}$ and obesity in the Qatari population. Additional data on dietary variables is needed in order to dissect the specific environmental contributions underlying these observations.

Taken together, these findings could partially explain the fluctuating success rate of studies attempting to replicate the associations between AMY1 CN and obesity in adults, as compared to more consistent results observed in pediatric cohorts, where lifestyle choices and age-dependent gene-environment interactions should be less pronounced.

In conclusion, here we investigate the association between adiposity traits and discrete copy number variation from WGS at highly polymorphic loci in the Qatari population. We demonstrated that within this single national population of the Middle East, the strength of genetic associations may differ significantly across individuals with different ethnic ancestries. To help explain this disparity, we also demonstrate the power of a large population biobank such as QBB, rich with genomic and phenotypic information, not only to drive discoveries of novel associations in a part of the world that is relatively understudied in the literature, but to provide plausible explanations for such discrepancies.

\section{METHODS}

\section{Cohort description}

The Qatar Biobank (QBB) is a national population-based medical health initiative started in 2012 with the aim of collecting samples and information on health and lifestyle from 60,000 Qatari adult nationals and long-term residents ${ }^{15}$. All subjects are enrolled in the Qatar Biobank via informed consent, and their genetic and phenotypic data made available for use in research studies. Anthropometrics details used in the current study, including BMI and body composition, were measured using the Seca stadiometer and Seca Bio Impedance Analysis (Seca GmbH and Co. KG, Hamburg, German) at Qatar Biobank facility at Hamad Medical City. Outliers (measurements greater than 3 standard deviations the dataset mean) were removed, and the data inverse-normal transformed.

The Qatar Biobank study was approved by the Qatar Biobank Institutional Review Board (IRB) Committee (IRB protocol E/2017/RESACC-0032/0002).

\section{Whole-genome sequencing data}

Subjects from the QBB were sequenced as part of the QGP. Whole-genome sequencing was performed on 2935 blood samples using Illumina HiSeq platform (150-bp paired-end) to an average coverage of 30x. Sequence reads were aligned to the Human Reference genome version GRCh37 using bwakit (v. 0.7.11).

\section{Amylase gene copy number estimation}

$A M Y 1$ and $A M Y 2$ CNs were estimated through sequencing coverage analysis by comparing the number of reads mapping to $A M Y 1$ and $A M Y 2$ genes to a reference local GC-matched and copy invariant region, as described in ref. ${ }^{1}$. Read counts were extracted from WGS bam files using samtools $^{38}$ (v. 1.6) with the command samtools view $-c$, defining the regions intervals with a corresponding bed file (coordinates based on GRCh37 genome assembly).
AMY1 corresponding region spans over $20 \mathrm{~kb}$ and includes the AMY1 gene plus two surrounding near-identical DNA sequences (chr1:104190000-104210000, 104227213-104247214 and 104284138104304150, respectively). Local $20 \mathrm{~kb}$ reference region intervals were: chr1:104059996-104070000 and chr1:104460001-104469995. No quality threshold was applied for filtering reads mapping to this region, being the repetitive region responsible for low local mapping quality scores, as suggested in ${ }^{1}$.

$A M Y 2 A C N$ was estimated counting reads mapping to the interval chr1:104153700-104161939, which includes exons 1-3 of AMY2A, and to its local copy-invariant comparator interval (chr1:104045000-104085000). A minimum mapping quality threshold of 20 was applied (samtools option: -q 20).

$A M Y 2 B C N$ was estimated counting reads mapping to the interval chr1:104114335-104135000 and to its local copy-invariant comparator interval (chr1:104000000-104100000 and chr1:104304000-104500000). A minimum mapping quality threshold of 20 was applied (samtools option: -q 20).

Local read density was calculated for $A M Y 1, A M Y 2 A, A M Y 2 B$, and their reference regions, dividing the absolute read count by the region size (e.g. the total number of reads mapping to $A M Y 1$ region divided by $40 \mathrm{k}$ ). Read densities were then normalized by their reference region read densities to obtain an estimate of their copy number state. Finally, raw $C N$ estimates were assigned to integers by conditional rounding. More in detail, $A M Y 2 A$ and $A M Y 2 B$ read densities were first rounded to the nearest integer. $A M Y 1$ read densities were then rounded to the nearest even number if $A M Y 2 A$ $\mathrm{CN}$ was even, and to the nearest odd number if $A M Y 2 A C N$ was odd.

Polychoric correlation between $\mathrm{CNs}$ was estimated using polycor $\mathrm{R}$ package (v. 0.7-10; polychor function).

\section{Evaluation of reads alignability at the amylase locus}

We assessed the rate of misalignment of sequences mapping to the AMY1 and $A M Y 2$ genes by evaluating the fraction of properly paired reads (i.e., read pairs mapping with correct orientation with respect to one another, and with expected insert size) mapping to either $A M Y 1$ or $A M Y 2 A$ having unique alignment. Alignability was estimated on the same genomic regions used for $\mathrm{CN}$ calling at $A M Y 1$ and $A M Y 2 A$. Within individual BAM files, uniquely mapping reads were defined based on the absence of the 'XA:Z:' and 'SA:Z:' tags (flagging secondary alignments and split reads, respectively) and the "**" score (flagging unmapped reads) in the mapping quality (MAPQ) field generated by bwa-mem (bwakit v. 0.7.11). Further, we evaluated the distribution of the mapping quality of reads aligned to the $A M Y 1$ and $A M Y 2 A$ genes.

\section{Validation of copy number estimates}

We used Droplet Digital ${ }^{\mathrm{TM}} \mathrm{PCR}^{39}$ (ddPCR, Bio-Rad) to experimentally validate $C N$ estimates at the $A M Y 1$ and $A M Y 2$ loci, following a previously published protocol ${ }^{3}$. PCR primers and fluorescent probes used in this study are reported in Supplementary Table 3. All probes targeting the AMY1 (two independent probes) and $A M Y 2 A$ (one probe) genes were labeled with FAM, whereas control probes with HEX. We digested the DNA with NHel restriction enzymes prior to amplification. The PCR reaction mix contained $0.25 \mathrm{ng} / \mathrm{ul}$ of DNA, $900 \mathrm{nM}$ of each primer (target and control), and $250 \mathrm{nM}$ of fluorescent probes. Droplet generation $(20,000)$ was done by QX200 Automated Droplet Generator, and the droplets were read by QX200 Droplet Reader. Droplet counts were analyzed using QuantaSoft software, and the copy number was estimated by dividing the number of FAMpositive droplets by the number of HEX-positive droplets (Supplementary Fig. 9). For each individual, the reported copy number represents an average of three independent replicates. Given that $A M Y 1$ and $A M Y 2 A$ share odd/even parity, we checked the correctness of the $A M Y 1 \mathrm{CN}$ call using the $A M Y 2 A$ call, as done in ref. ${ }^{3}$. For a given sample, if both $A M Y 1$ calls obtained from two independent probes were concordant, they were averaged. If only one was concordant, only the concordant AMY1 genotype was used. If both calls were discordant, they were averaged.

A further Independent in silico validation of $A M Y 1 C N$ in the Qatari population was performed using AMYCNE $^{18}$ (release: 2020-03-18) which was developed particularly for genes harboring more than four copies, and its accuracy in estimating $A M Y 1 \mathrm{CN}$ was assessed by the authors using ddPCR, making it particularly suitable for identifying $A M Y 1 \mathrm{CN}$ variations. Copy number at $A M Y 1$ was estimated using region files provided by AMYCNE and BAM coverage files obtained using TIDDIT $^{40}$. 


\section{Loss-of-function and missense variants at the $A M Y 1$ gene}

Coding-region variants at the AMY1 gene were annotated using SNPeff ${ }^{41}$ which implements coding effect prediction of genetic variants. Stop gained, frameshift, missense variants or inframe deletions were considered as high-impact variants.

\section{$A M Y 1, A M Y 2 A$, and adiposity traits association testing in the Qatari population}

We tested the association between amylase genes $\mathrm{CNs}$ and adiposity traits in 2935 individuals from the QBB. Association testing was carried out using PopPAnTe $^{19}$ (v. 1.0.2) which uses a variance component framework to model the resemblance among related individuals. Subjects showing zero $\mathrm{CN}$ at $A M Y 1$ were discarded from the analysis, and rare $A M Y 1 \mathrm{CNs}$ larger than 14 copies were set to 14 . Sex, age at the sample collection, and the first 10 PCs from genome-wide data were included as covariates. The phenotypic covariance matrix between subjects was modeled using the matrix of the kinship between each pair of individuals, as evaluated by $\mathrm{PLINK}^{42}$. Indeed, the Qatari population is an isolated inbred population characterized by a large number of consanguineous families ${ }^{43}$. Pairs of individuals having a kinship lower than 0.05 were considered as unrelated (i.e., their kinship was considered to be zero).

We derived the Bonferroni-adjusted threshold for statistical significance by dividing a conventional alpha value of 0.05 by the number of tests performed, considering both the number of outcome variables $(N=3)$ and the number of predictors tested (i.e., $A M Y 1$ and $A M Y 2 A ; N=2$ ).

\section{$A M Y 1$ and adiposity traits association testing in Arabs and Persians from Qatar}

To identify the ethnicity of each of the 2935 participants from the QBB, we leveraged genotypes at 48 ancestry-informative single-nucleotide polymorphisms (SNPs) from the WGS data to differentiate the three major Qatari subpopulations (Bedouin Arabs, Persians, East Africans) as described in refs. ${ }^{20,21}$. Individuals whose majority ancestry did not belong to these three major ethnicities were considered admixed.

Subsequently, we sought for $A M Y 1$ association with adiposity traits using PopPAnTe (v. 1.0.2), in 948 Persians and 1518 Arabs from the QBB. Subjects showing zero $\mathrm{CN}$ at $A M Y 1$ were discarded from the analysis, and rare $A M Y 1$ CNs larger than 14 copies were set to 14 . Sex, age at the sample collection, and the first 10 PCs from genome-wide data were included as covariates.

The Bonferroni-adjusted threshold for statistical significance was obtained by dividing a conventional alpha value of 0.05 by the number of tests performed, considering both the number of outcome variables ( $N$ $=3)$ and of groups tested $(N=2)$.

\section{Arabs and Persians clustering based on genotypes at the amylase region}

To verify whether some degree of admixture at the amylase genomic region was not properly captured by the ethnicity-informative SNPs ${ }^{20,21}$ used to assign subjects to the different ethnic groups, we carried out PCA on 6,229 SNPs and indels located within a 5-MB window surrounding AMY1 (chr1:99,159,954-109,301,314; Supplementary Fig. 4). PCA was performed using PLINK (v. 1.9) on LD-pruned high-quality variants (MAF $>0.01$, genotyping rate $>0.9$, HWE deviation $P>10^{-6}$ ). LD pruning was carried on with PLINK (option: --indep-pairwise) using the following parameters: window size of $50-\mathrm{kb}$, shift of 5 variants, and $r^{2}$ threshold of 0.05 . This analysis highlighted the presence of a small number of outliers $\left(N_{\text {Arabs }}=\right.$ $75, N_{\text {Persians }}=34$, Supplementary Fig. 4). Therefore, to rule out the possibility that these individuals could possibly be skewing the association signal in our primary analysis, we removed them from our dataset, and reassessed the association between $A M Y 1 \mathrm{CN}$ and adiposity traits using PopPAnTe (v. 1.0.2), and including sex, age, and the first 10 PCs from genome-wide data as covariates.

\section{Case/control $A M Y 1$ association with obesity in Arabs from Qatar} Association of $A M Y 1$ CNs with obesity was assessed in Arabs from the QBB by logistic regression using R (Ime4 package, v. 1.1-21). Individuals with $\mathrm{BMI} \geq 30 \mathrm{~kg} / \mathrm{m}^{2}$ were considered as obese, whereas subjects with $\mathrm{BMI}<$ $25 \mathrm{~kg} / \mathrm{m}^{2}$ were treated as controls. Rare AMY1 CNs larger than 14 were set to 14 . Sex, age at the sample collection and the first 10 PCs from genomewide data were included as covariates in the analysis.

\section{AMY1 association with dietary restraint}

In order to explore whether ethnic-specific behavioral variations underlie inter-population variability in $A M Y 1$ association with adiposity, we sought association between $A M Y 1$ copy number and dietary restraint, collected through self-administered questionnaires as part of the QBB initiative.

Association of self-reported dietary restraint with $A M Y 1 \mathrm{CNs}$ was evaluated in overweight subjects $\left(B M I \geq 25 \mathrm{~kg} / \mathrm{m}^{2}\right.$ ) of Persians or Arabs ancestry from the QBB by logistic regression in $\mathrm{R}$ (Ime4 package, v. 1.1-21), including age, sex, BMI, and ethnicity as covariates. Rare AMY1 copy numbers larger than 14 were set to 14 .

\section{Dissecting the confounding effect of ethnicity}

To dissect the effect of ethnicity in our analyses, we evaluated the association between the first principal component (PC1) values and AMY1 $\mathrm{CNs}$, as well as adiposity traits. We also investigated the association between AMY1 CNs and adiposity traits, as well as the tendency towards dietary restraint behaviors, in subsets of individuals of Arab or Persians ancestry, binned according to their PC1 values (Supplementary Fig. 10). More in detail, we generated 10 sliding windows, each including 750 Arab individuals (with an overlap of 85 individuals) along with the first principal component in Arabs. Similarly, three sliding windows, each including 750 Persian individuals (with 85 individuals of overlap) were generated along with the first principal component in Persian. Within each window, we tested the association between $A M Y 1 \mathrm{CN}$ and BMl, total and trunk fat mass percentages using linear regression (stats $\mathrm{R}$ package, v. 3.6). Outliers (measurements greater than three standard deviations the dataset mean) were removed, and the data inverse normal transformed. Age and sex were included as covariates. Association with dietary restraint was assessed using logistic regression (stats $\mathrm{R}$ package, v. 3.6), and adjusting for sex, age, and BMI.

Heritability of the adiposity traits was estimated using PopPAnTe ( $v$. 1.0.2), accounting for age and sex.

\section{Reporting summary}

Further information on research design is available in the Nature Research Reporting Summary linked to this article.

\section{DATA AVAILABILITY}

The anonymized study participants information on ancestry, estimated $A M Y 1$ and AMY2 copy numbers, BMI, total and trunk fat percentages (age and sex-adjusted standardized residuals) are available as Supplementary Data 1. Raw data should be requested through filling the access application at website www.qatarbiobank.org.qa and submitted to the research access office accessofficeqbb@qf.org.qa. Requests are reviewed and approved by QBB IRB and Access Committee.

\section{CODE AVAILABILITY}

Analyses were carried out using open-source published software which are referenced in the manuscript.

Received: 19 June 2020; Accepted: 24 December 2020; Published online: 09 February 2021

\section{REFERENCES}

1. Carpenter, D. et al. Obesity, starch digestion and amylase: association between copy number variants at human salivary (AMY1) and pancreatic (AMY2) amylase genes. Hum. Mol. Genet. 24, 3472-3480 (2015).

2. Carpenter, D., Mitchell, L. M. \& Armour, J. A. L. Copy number variation of human AMY1 is a minor contributor to variation in salivary amylase expression and activity. Hum. Genomics 11, 1-6 (2017).

3. Usher, C. L. et al. Structural forms of the human amylase locus and their relationships to SNPs, haplotypes and obesity. Nat. Genet. 47, 921-925 (2015).

4. Groot, P. C., Mager, W. H. \& Frants, R. R. Interpretation of polymorphic DNA patterns in the human a-amylase multigene family. Genomics 10, 779-785 (1991).

5. Perry, G. H. et al. Diet and the evolution of human amylase gene copy number variation. Nat. Genet. 39, 1256-1260 (2007).

6. Falchi, M. et al. Low copy number of the salivary amylase gene predisposes to obesity. Nat. Genet. 46, 492-497 (2014).

7. Yong, R. Y. Y. et al. Complex copy number variation of $A M Y 1$ does not associate with obesity in two east asian cohorts: HUMAN MUTATION. Hum. Mutat. 37, 669-678 (2016). 
8. Shwan, N. A. A. \& Armour, J. A. L. No evidence for association of BMI with salivary amylase gene copy number in the UK 1958 birth cohort: AMY1 copy number and BMI. Obesity 27, 1533-1538 (2019).

9. Bonnefond, A. et al. Relationship between salivary/pancreatic amylase and body mass index: a systems biology approach. BMC Med. 15, 37 (2017).

10. Mejía-Benítez, M. A. et al. Beneficial effect of a high number of copies of salivary amylase AMY1 gene on obesity risk in Mexican children. Diabetologia 58, 290-294 (2015)

11. Marcovecchio, M. L. et al. Low AMY1 gene copy number is associated with increased body mass index in prepubertal boys. PLOS ONE 11, e0154961 (2016).

12. Viljakainen, $\mathrm{H}$. et al. Low copy number of the AMY1 locus is associated with earlyonset female obesity in Finland. PLOS ONE 10, e0131883 (2015).

13. Rukh, G., Ericson, U., Andersson-Assarsson, J., Orho-Melander, M. \& Sonestedt, E. Dietary starch intake modifies the relation between copy number variation in the salivary amylase gene and BMI. Am. J. Clin. Nutr. 106, 256-262 (2017).

14. Heianza, Y. et al. Starch digestion-related amylase genetic variant affects 2-year changes in adiposity in response to weight-loss diets: the POUNDS lost trial. Diabetes 66, 2416-2423 (2017).

15. Al Kuwari, $\mathrm{H}$. et al. The Qatar Biobank: background and methods. BMC Public Health 15, 1208 (2015)

16. Santos, J. L. et al. Copy Number Polymorphism of the Salivary Amylase Gene: Implications in Human Nutrition Research. J. Nutr. Nutr. 5, 117-131 (2012).

17. Genome Aggregation Database Consortium. et al. The mutational constrain spectrum quantified from variation in 141,456 humans. Nature 581, 434-443 (2020).

18. Eisfeldt, J., Nilsson, D., Andersson-Assarsson, J. C. \& Lindstrand, A. AMYCNE: Confident copy number assessment using whole genome sequencing data. PLOS ONE 13, e0189710 (2018)

19. Visconti, A. et al. PopPAnTe: population and pedigree association testing for quantitative data. BMC Genomics 18, (2017).

20. Fakhro, K. A. et al. Copy number variations in the genome of the Qatari population. BMC Genomics 16, (2015).

21. Rodriguez-Flores, J. L. et al. Exome sequencing identifies potential risk variants for mendelian disorders at high prevalence in Qatar. Hum. Mutat. 35, 105-116 (2014).

22. Hruby, A. \& Hu, F. B. The epidemiology of obesity: a big picture. PharmacoEconomics 33, 673-689 (2015).

23. Al-Thani, M. H. et al. Prevalence and determinants of metabolic syndrome in Qatar: results from a National Health Survey. BMJ Open 6, e009514 (2016).

24. Tomei, S. et al. Obesity susceptibility loci in Qataris, a highly consanguineous Arabian population. J. Transl. Med. 13, 119 (2015).

25. Hebbar, P. et al. The TCN2 variant of rs9606756 [lle23Val] acts as risk loci for obesity-related traits and mediates by interacting with Apo-A1: TCN2 Variant as Risk Loci for Waist Circumference. Obesity 25, 1098-1108 (2017).

26. O'Beirne, S. L. et al. Type 2 diabetes Risk Allele Loci in the Qatari population. PLoS ONE 11, e0156834 (2016).

27. Al Muftah, W. A. et al. Epigenetic associations of type 2 diabetes and BMI in an Arab population. Clin. Epigenet. 8, 13 (2016).

28. Mandel, A. L., Peyrot des Gachons, C., Plank, K. L., Alarcon, S. \& Breslin, P. A. S. Individual differences in AMY1 gene copy number, salivary a-amylase levels, and the perception of oral starch. PLoS ONE 5, e13352 (2010).

29. Aji, G. K., Warren, F. J. \& Roura, E. Salivary a-amylase activity and starch-related sweet taste perception in humans. Chem. Senses 44, 249-256 (2019).

30. León-Mimila, P. et al. Low Salivary Amylase Gene (AMY1) copy number is associated with obesity and gut prevotella abundance in Mexican children and adults. Nutrients 10, 1607 (2018)

31. Poole, A. C. et al. Human salivary amylase gene copy number impacts oral and gut microbiomes. Cell Host Microbe 25, 553-564.e7 (2019).

32. Nakajima, K. \& Oda, E. Ketonuria may be associated with low serum amylase independent of body weight and glucose metabolism. Arch. Physiol. Biochem. 123, 293-296 (2017)

33. Arredouani, A. et al. Metabolomic profile of low-copy number carriers at the salivary a-amylase gene suggests a metabolic shift toward lipid-based energy production. Diabetes 65, 3362-3368 (2016).

34. Hjorth, M. F. et al. Pre-treatment microbial Prevotella-to-Bacteroides ratio, determines body fat loss success during a 6-month randomized controlled diet intervention. Int. J. Obes. 42, 580-583 (2018).

35. Hjorth, M. F. et al. Prevotella-to-Bacteroides ratio predicts body weight and fat loss success on 24-week diets varying in macronutrient composition and dietary fiber: results from a post-hoc analysis. Int. J. Obes. 43, 149-157 (2019).

36. Badran, M. \& Laher, I. Obesity in Arabic-speaking Countries. J. Obes. 2011, 1-9 (2011).

37. Al-Akl, N., Thompson, R. I. \& Arredouani, A. High plasma salivary a-amylase, but not high AMY1 copy number, associated with low obesity rate in Qatari adults: cross-sectional study. Sci. Rep. 10, 1-11 (2020).
38. $\mathrm{Li}, \mathrm{H}$. et al. The sequence alignment/map format and SAMtools. Bioinformatics $\mathbf{2 5}$ 2078-2079 (2009).

39. Hindson, B. J. et al. High-throughput droplet digital PCR system for absolute quantitation of DNA copy number. Anal. Chem. 83, 8604-8610 (2011).

40. Eisfeldt, J., Vezzi, F., Olason, P., Nilsson, D. \& Lindstrand, A. TIDDIT, an efficient and comprehensive structural variant caller for massive parallel sequencing data. F1000Research 6, 664 (2017).

41. Cingolani, P. et al. A program for annotating and predicting the effects of single nucleotide polymorphisms, SnpEff: SNPs in the genome of Drosophila melanogaster strain w1118; iso-2; iso-3. Fly 6, 80-92 (2012).

42. Chang, C. C. et al. Second-generation PLINK: rising to the challenge of larger and richer datasets. GigaScience 4, s13742-015 (2015).

43. Bener, A. \& Alali, K. A. Consanguineous marriage in a newly developed country: the Qatari population. J. Biosoc. Sci. 38, 239-246 (2006).

\section{ACKNOWLEDGEMENTS}

We would like to thank the Qatar Biobank and the Qatar Genome Programme for providing access to the phenotyping and genomic datasets for this study. This study was funded in part by the Qatar National Research Fund's (QNRF) and Qatar Genome Programme's (QGP), Path towards Precision Medicine award (PPM1-1229-15002) and Sidra Medicine's Precision Medicine Program (SDR100011). This research received no specific grant from any funding agency in the commercial sector. We thank John Armour for his advice and suggestions on the assessment of amylase genes copy number in WGS data. We are grateful to all the participants of this study.

\section{AUTHOR CONTRIBUTIONS}

N.R., M.F., A.V., and K.A.F. designed the study. N.R., E.A., N.S., and A.V. conducted the computational experimentation. W.A. and S.S.P. conducted experimental validation N.R., M.F., E.A., and K.A.F. interpreted the results. N.R., A.V., A.S.A.A., M.F., and K.A.F. drafted the manuscript. All authors read and approved the final draft of the manuscript.

\section{FUNDING}

Open access funding provided by the Qatar National Library.

\section{COMPETING INTERESTS}

The authors declare no competing interests.

\section{ADDITIONAL INFORMATION}

Supplementary information The online version contains supplementary material available at https://doi.org/10.1038/s41525-021-00170-3.

Correspondence and requests for materials should be addressed to K.A.F.

Reprints and permission information is available at http://www.nature.com/reprints

Publisher's note Springer Nature remains neutral with regard to jurisdictional claims in published maps and institutional affiliations.

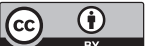

Open Access This article is licensed under a Creative Commons Attribution 4.0 International License, which permits use, sharing, adaptation, distribution and reproduction in any medium or format, as long as you give appropriate credit to the original author(s) and the source, provide a link to the Creative Commons license, and indicate if changes were made. The images or other third party material in this article are included in the article's Creative Commons license, unless indicated otherwise in a credit line to the material. If material is not included in the article's Creative Commons license and your intended use is not permitted by statutory regulation or exceeds the permitted use, you will need to obtain permission directly from the copyright holder. To view a copy of this license, visit http://creativecommons. org/licenses/by/4.0/.

(c) The Author(s) 2021 